\title{
Miocarditis en Niños con Fiebre por Dengue Hemorrágico en un Hospital Universitario de Colombia
}

\section{Myocarditis in children affected by dengue hemorrhagic fever in a teaching hospital in Colombia}

\author{
Doris M. Salgado, Cesar A. Panqueba, Dolly Castro, Martha R.Vega \\ y Jairo A Rodríguez
}

Facultad de Salud, Universidad Surcolombiana. Neiva, Colombia. domasa159@yahoo.com, docastro@usco.edu.co,martharociovega@hotmail.com,jrodriguez@usco.edu.co

Recibido 24 Septiembre 2008/Enviado para Modificación 17 Mayo 2009/Aceptado 16 Julio 2009

\section{RESUMEN}

Objetivo Determinar la frecuencia de miocarditis en niños con fiebre por Dengue hemorrágico-FDH, en el Huila, Colombia.

Materiales y Métodos Se realizó un estudio descriptivo de observación prospectiva, entre Marzo del 2005 y Octubre de 2006. Se observaron 102 pacientes con diagnóstico de $\mathrm{FDH}$, basado en los criterios de la OMS, para identificar la frecuencia de miocarditis. Resultados Un $10,7 \%$ de los niños presentaron miocarditis. Solo un caso con miocarditis fue clasificado como dengue II; los otros diez casos fueron dengue III y IV. La diferencia en la presentación de miocarditis en los estadíos más severos de dengue fue estadísticamente significativa, con un $\mathrm{p}=0.0004$. Diez de los pacientes tuvieron miocarditis que respondió al soporte inotrópico temprano. En el electrocardiograma (EKG), dos pacientes presentaron taquiarritmias, uno tipo supraventricular y otro ventricular, el resto mostró bradicardia sinusal con trastornos de repolarización y bajos voltajes. El ecocardiograma se realizó en 50 \% de los niños, encontrándose derrame pericárdico grado $-1 l$ en dos pacientes y uno con la fracción de eyección muy disminuida. La estancia promedio fue de 7 días. Un paciente presentó desenlace fatal.

Conclusión La presentación de miocarditis alerta sobre la presencia de disfunción miocárdica como una patología a considerarse en pacientes con $\mathrm{FDH}$.

Palabras Clave: Dengue, fiebre dengue hemorrágica, miocarditis (fuente: DeCS, BIREME).

\section{ABSTRACT}

Objective To determining myocarditis frequency in children suffering from DHF in the Huila region of Colombia.

Methods This was a descriptive and prospective study which was carried out between March 2005 and October 2005 involving 102 patients suffering from DHF, based on WHO criteria. These children were followed-up to identify signs of myocarditis. 
Results $10.7 \%$ of the children had signs of myocarditis; one of them was classified as having dengue II and the other ten were classified as suffering form dengue III and IV. This difference was statistically significant $(p=0.0004)$. 10 patients had fulminant myocarditis and required early inotropic support. Two patients had tachyarrythmias in the EKG (one SV and one ventricular); the others had sinusal bradichardia and low voltages. Echocardiograms were perfomed on $50 \%$ of the children, revealing pericardial leakage grade I and II in two patients, one with very diminished EF and AF. Average hospital stay was 7 days. 10 had satisfactory outcome whereas one died.

Conclusion This series of cases in children reinforces the association between myocarditis and dengue viral infection.

Key Words: Dengue, dengue hemorrhagic fever, myocarditis (source: MeSH, NLM).

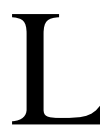

a fiebre dengue hemorrágica (FDH) es considerada la enfermedad viral transmitida por vectores más importante a nivel mundial, con 2,5 billones de habitantes susceptibles de padecerla, con un incremento en la incidencia registrada en las Américas en los últimos años y la presencia de hiperendemicidad viral similar a lo observado en el sudeste asiático (1).

El virus dengue (VD) está compuesto por cuatro serotipos (DEN1, DEN2, DEN3, y DEN4); se ha comprobado la circulación de todos los serotipos en el continente americano.

En los últimos años, se ha hablado de manifestaciones clínicas atípicas $(2,3)$ relacionadas con el incremento en los casos y la variedad de cepas circulantes, que ha permitido reconocer un comportamiento clínico no usual, con compromiso de órganos como hígado, tracto gastrointestinal, sistema nervioso central y corazón entre otros (4-6), lo que favorece presentaciones clínicas complicadas e incluso fatales $(7,8)$.

La miocarditis es una entidad eminentemente viral siendo los enterovirus, los principales agentes etiológicos (9). En algunos pacientes infectados por VD se ha descrito un efecto cronotrópico negativo sobre el miocardio (10).

Se propone una respuesta inmunológica asociada con la mayor lesión del miocardio a través de la cascada inflamatoria iniciada por el sistema monocitomacrófago con liberación de IL-1, IL-6 y especialmente el TNF por su efecto cronotrópico negativo (11); considerando este tipo de compromiso en la FDH donde hay liberación masiva de citoquinas inflamatorias, sumado a la existencia de un probable mimetismo molecular, producido por reacción cruzada entre péptidos virales con antígenos normales de diferentes tejidos, entre ellos el miocárdico, llevan a una actividad citotóxica y lisis celular ( miocito) $(9,12)$. 
Los primeros reportes del compromiso cardiaco en Dengue son referidos por Nagaratman (13) con un caso de miopericarditis en 1972. Más tarde se comenzó a hablar de cambios electrocardiográficos acompañando la enfermedad siendo Yusoff quien en su artículo de 1993 hace una detallada descripción de estos (14).

En las Américas, Martínez en Cuba (15), hace las primeras referencias al compromiso miocárdico en niños en 1995; pero son Wali y Kabra en 1998 en India quienes presentan el cuadro de disfunción miocárdica, claramente definido y de evolución favorable con imágenes y estudios de laboratorio (16,17). En el año 2003 aparece el artículo de Veloso en Brasil, presentando un caso de arritmia (18), simultáneamente en el sudeste asiático se insiste en esta presentación gracias a las descripciones de Khongphatthanayothin, y Promphan-bradiarritmias $(10,19)$.

Malaguive en el 2004, señala al compromiso miocárdico como una de las manifestaciones atípicas en la presentación del dengue (2). En India, se considera como causa de choque refractario y se indica por Ramjit $S$, el manejo agresivo hemodinámico en la unidad de cuidados intensivos (20). En 2007 Gulati realizó una compilación de manifestaciones inusuales asociadas al dengue en las que se incluye la miocarditis (3).

En Colombia entre 2005 y 2006, se presentan dos reportes refiriendo disfunción miocárdica en dengue: $8 \%$ (21) y $13 \%$ (22).

El objetivo de este estudio es establecer la frecuencia de miocarditis asociada al dengue en los pacientes hospitalizados en la unidad de cuidados intensivos pediátricos (UCIP) del Hospital Universitario Hernando Moncaleano Perdomo de Neiva, Colombia (HUHMP).

\section{MATERIALES Y MÉTODOS}

Este es un estudio prospectivo descriptivo, que incluyó todos los niños con edad menor o igual a 13 años admitidos al servicio de Pediatría del HUHMP, durante el período comprendido entre marzo 2005 y octubre 2006, con diagnóstico de FDH, estadios I a IV de acuerdo con los criterios establecidos por la Organización Mundial de la Salud, confirmado por serología (IgM específica contra el virus, PanBio) o por RT-PCR en el Instituto Nacional de Salud (Bogotá, Colombia) y de miocarditis, siguiendo la clasificación establecida en la Guías de Miocarditis 2006 (Tabla 1). 
Tabla 1. Clasificación clínica de miocarditis

\begin{tabular}{lll}
\hline Miocarditis fulminante & $\begin{array}{l}\text { Miocarditis subaguda o } \\
\text { crónica }\end{array}$ & Miocardiopatía dilatada \\
\hline Curso viral corto & $\begin{array}{l}\text { Pródromo viral } \\
\text { Falla cardiaca }\end{array}$ & $\begin{array}{l}\text { Falla cardiaca congestiva } \\
\text { Cardiomegalia en los Rx } \\
\text { de tórax }\end{array}$ \\
$\begin{array}{l}\text { Muerte súbita } \\
\text { Disfunción ventricular } \\
\text { izquierda }\end{array}$ & $\begin{array}{l}\text { Disfunción ventrículo } \\
\text { izquierdo }\end{array}$ \\
$\begin{array}{l}\text { Tamaño cardiaco normal } \\
\text { Disfunción ventricular }\end{array}$ & & Historia Familiar \\
izquierda & & \\
\hline Tomada y adaptada de (9) & &
\end{tabular}

A algunos pacientes con sospecha clínica de miocarditis se le realizaron las siguientes pruebas diagnósticas, previo consentimiento informado, para identificar la miocarditis: ecocardiograma, creatín fosfoquinasa fracción MB (CPK MB) mediante la técnica de inmunoinhibición usando un kit comercial (Hitachi 911, Roche, Manheim, Germany).

Los datos de los pacientes se llevaron a una base de datos en Epi-info versión 3.3.2 de 2005 y se utilizó STATA 9.0 para el análisis de los casos de miocarditis. Para el cruce de variables o correlación se utilizó la prueba de Chicuadrado. El estudio fue presentado y aprobado por el comité de ética del Hospital.

\section{RESULTADOS}

De 102 niños con FDH, el $18 \%$ (19 niños) se clasificaron en estadio I y II, el $78 \%$ (79 niños) estadio III; el 4 \% (4 niños) en estadio IV. El diagnóstico de miocarditis se hizo en 11 pacientes, de los cuales diez se clasificaron como dengue III y IV. Esta diferencia en la presentación de miocarditis en relación a la severidad del dengue es estadísticamente significativa $(\mathrm{p}=0.0004)$.

La Tabla 2 muestra los resultados clínicos y de laboratorio de los niños incluidos en el estudio encontrándose que el promedio de edad estuvo alrededor de los 6 años; no hubo diferencia respecto al género; fiebre de 5 días de evolución, cefalea $(38,2 \%)$, vómito $(57,8 \%)$, dolor abdominal $(61,8 \%)$, mialgias $(35,3 \%)$, sangrado $(39,2 \%)$ y disnea $(7,8 \%)$.

$\mathrm{Al}$ examen físico el $36 \%$ de los pacientes tuvo cifras de tensión arterial sistólica por debajo del percentil 5, que correspondió al estadio de dengue IV, 
bradicardia en 9 de los 11 casos, 79,4\% con hepatomegalia y signos de fuga vascular en el $90 \%$ (Tabla 3).

En cuanto a los paraclínicos, se encontró el hematocrito al ingreso normal (36,4 en promedio con un rango de 27 a 45), recuento plaquetario (47 000 en promedio con rango de 11000 a 86 000), pruebas de coagulación que a pesar de encontrarse alteradas por aumento en su valores respecto a lo normal, no se pudo relacionar con sangrados importantes dado que los pacientes solo presentaron petequias y hematomas leves.

Tabla 2. Hallazgos clínicos y de laboratorio de los pacientes con miocarditis Dengue $(n=11)$

\begin{tabular}{lcc}
\hline \multicolumn{1}{c}{ Parámetro } & Mediana & $\begin{array}{c}\text { Rango intercuartílico } \\
(25 \%, 75 \%)\end{array}$ \\
\hline Edad (meses) & 72 & $(13,120)$ \\
Días de fiebre & 5 & $(4,5)$ \\
Días de estancia & 6 & $(3,8)$ \\
Presión arterial sistólica (mmHg) & 96 & $(87,100)$ \\
Presión de pulso (mmHg) & 30 & $(29,36)$ \\
Frecuenta cardiaca ( por minuto) & 67 & $(61,84)$ \\
Derrame pleural (porcentaje) & 35 & $(0,50)$ \\
Plaquetas (por mm $\left.^{3}\right)$ & 45000 & $(29000,74000)$ \\
Hematocrito (porcentaje) & 38 & $(32,40)$ \\
TP (segundos) & 15 & $(14,23)$ \\
TPT (segundos) & 45 & $(32,62)$ \\
AST(Ul/mL) & 115 & $(71,200)$ \\
ALT (Ul/mL) & 50 & $(27,64)$ \\
\hline
\end{tabular}

TP: tiempo de protrombina; TPT: tiempo parcial de tromboplastina; AST: aspartato amino transferasa; ALT: alanino amino transferasa

Tabla 3. Hallazgos electro y ecocardiográficos en pacientes con miocarditis dengue

\begin{tabular}{lc}
\multicolumn{1}{c}{ Hallazgo } & $N^{\circ}$ pacientes \\
\hline Bradicardia & $9 / 11$ \\
Taquicardia & $2 / 11$ \\
TSV & $1 / 11$ \\
TV & $1 / 11$ \\
Alteraciones en el ST-T & $7 / 11$ \\
Derrame pericárdico & $5 / 7$ \\
Disfunción ventricular & $2 / 7$ \\
\hline TSV= taquicardia supraventricular TV-taquicardia ventricular;
\end{tabular}

$T S V=$ taquicardia supraventricular; $T V=$ taquicardia ventricular; $\mathrm{ST}=$ segmento $\mathrm{ST}, \mathrm{T}=$ onda $\mathrm{T}$ en Electrocardiograma

Las pruebas hepáticas se encontraron incrementadas, especialmente la aspartato amino-transferasa (AST) cuyo valor estuvo por encima de 10 veces lo 
normal; la CPK-MB tuvo un valor promedio de 169 UI/dL, además, se encontró una correlación significativa $(\mathrm{p}<0.0001)$ entre estas dos pruebas bioquímicas en los niños con miocarditis (Figura 1). A cinco pacientes a quienes se les tomó la Creatinfosfokinasa fracción MB (u/l), AST: aspartato transferasa (u/l) séricas se les realizó un test de correlación.

Figura 1. Correlación de la CPK-MB y la AST en pacientes con dengue hemorrágico

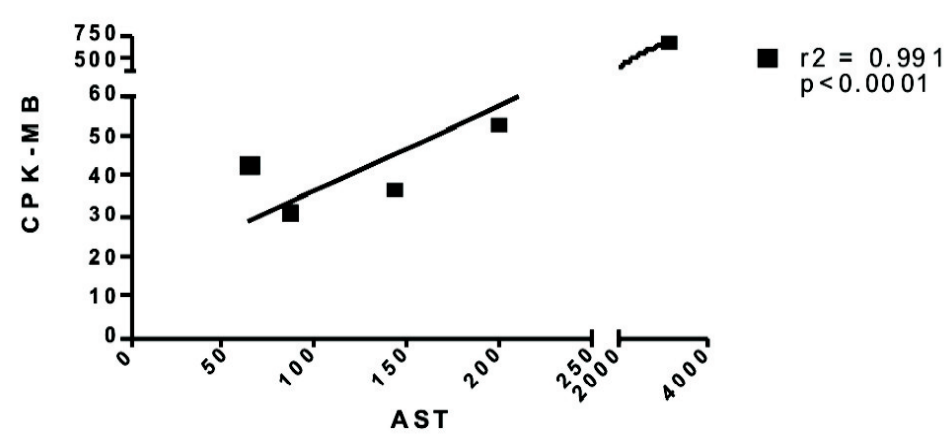

A todos los pacientes se les practicó electrocardiograma, encontrando en 9 deellos bradicardia sinusal, dos presentaron taquiarritmias, uno de tipo taquicardia supraventricular y el otro de tipo ventricular; 7 con trastornos de la repolarizacion y bajos voltajes. El ecocardiograma se realizó a 7 niños demostrando en promedio una leve disminución en la fracción de eyección, 5 con leve derrame pericárdico y franca disfunción diastólica en dos (Tabla 3).

De acuerdo a los criterios de inclusión para miocarditis, 10 pacientes fueron clasificados como miocarditis fulminante con respuesta favorable al soporte inotrópico, el cual fue recibido por la totalidad de los pacientes incluyendo uno o más de estos medicamentos. Un paciente cursó con miocarditis fulminante y desenlace fatal.

\section{DISCUSIÓN}

Kabra y colaboradores, describieron la presentación de disfunción miocárdica en $9(16,5 \%)$ de 54 pacientes con FDH, determinada ecocardiográficamente (16). Wali JP, en un estudio de 17 pacientes con infección por DEN 2, mostró disminución en la función cardiaca, hipoquinesia global y una baja fracción de eyección ventricular (40\%) en 7 pacientes (17), la cual puede estar asociada con una precarga disminuida que explica una frecuencia cardiaca anormal y alteración de la función ventricular izquierda (19). 
Todas estas descripciones mostraban miocardiopatía de buen pronóstico con alteraciones de la conducción (15); En este estudio, se encontraron importantes alteraciones en el ritmo cardiaco como fenómeno prevalente en 9 de los 11 pacientes, similar a lo descrito anteriormente, pero que necesitaron apoyo inotrópico con respuesta favorable, sin embargo, en publicaciones recientes este compromiso se describe como una miocarditis aguda fulminante, potencialmente fatal, en pacientes que ingresan con choque a las unidades de cuidados intensivos (2326). Este hallazgo es similar a lo encontrado en el paciente que falleció en este estudio.

En una serie previa de casos de mortalidad por VD en el Hospital Universitario de Neiva ya se describe a la miocarditis como la principal causa de muerte en niños con FDH (27); manifestación esta, severa y de mal pronóstico en infección por VD.

En general, a la fecha existen dificultades para la definición de caso confirmado de miocarditis, cuyos criterios aún se encuentran en discusión. Aunque para el 2006, en el consenso internacional para miocarditis viral se plantea un espectro de enfermedad que va desde cuadro clínicos agudos fulminantes, pasando por miocarditis subaguda o crónica hasta la miocardiopatía dilatada basados en aspectos clínicos, de laboratorio, hallazgos histológicos (clasificación de Dallas) y evidencia de infección viral (9).

En esta cohorte de pacientes el diagnóstico de miocarditis se hizo en el $10.7 \%$ (11 de 102) considerando hallazgos clínicos como choque cardiogénico, alteraciones en el EKG, ecocardiograma y evidencia de infección viral con IgM positiva y RT-PCR para VD en tres casos, dos DEN 3, uno de los casos fue el de el paciente fallecido y un caso DEN 2.

Los escolares se convirtieron en el grupo de mayor presentación de la patología (69m), a diferencia de las otras miocarditis virales con aparición temprana $(4,5$ días) de los signos de miocarditis en el curso del cuadro viral, con choque y trastornos del ritmo predominando la bradicardia, requiriendo soporte inotrópico e ingreso a la UCIP.

El estudio EKG mostró alteración en todos los casos, el ecocardiograma se realizó posterior al inicio de los inotrópicos lo que explicaría el hallazgo de fracciones de eyección y de acortamiento normales. A pesar de que estas dos herramientas son descritas como menos específicas en el diagnóstico de miocarditis, son de gran utilidad, en el caso de dengue especialmente el EKG, cuyas alteraciones 
han sido reportadas de forma constante en la literatura (taquicardia sinusal que persiste a pesar de la corrección del choque, bradicardia, complejos QRS de bajo voltaje, aplanamiento del segmento ST y onda $\mathrm{T}$ y arritmias) y en sitios de alta endemicidad puede constituirse como primer estudio de tamizaje.

Las enzimas cardiacas fueron realizadas a 6 pacientes con valores por encima de lo normal especialmente en el paciente con miocarditis fulminante fatal, sugiriendo necrosis miocárdica. En esta serie, los pacientes se clasificaron todos como miocarditis fulminante considerando la rápida instauración del cuadro clínico, con choque cardiogénico requiriendo soporte inotrópico, alteraciones del ritmo cardiaco y resolución rápida favorable en el $90 \%$ de los casos que coincide con lo descrito en otros estudios (16-17).

Los niños con miocarditis ingresaron con diagnóstico de choque FDH en su mayoría, con una correlación entre miocarditis y severidad por FDH con significancia estadística (p 0.0004), que permite plantear la importancia de esta manifestación inusual y obliga a la atención de estos pacientes en UCIP con intervenciones medicamentosas agresivas que garanticen la supervivencia de los niños.

El compromiso miocárdico descrito durante la infección por $\mathrm{VD}$, generalmente es benigno y transitorio, se caracteriza por bradicardia o alteraciones en el ritmo que se presentan durante la fase de recuperación de la enfermedad, sin embargo, los pacientes en este estudio mostraron signos de miocarditis principalmente en la fase aguda; en promedio al quinto día, cuando la viremia aún se encuentra alta, lo cual podría plantear un rol directo del virus en la patogénesis con efecto citolítico, aspecto que debe seguir siendo investigado, por que a la fecha solo se ha demostrado replicación viral en células musculares esqueléticas. El porcentaje de letalidad $(1 \%)$ en esta serie muestra la importancia que puede tener esta forma de presentación como causa de mortalidad por dengue.

Existió dificultad para la toma de ecocardiograma de ingreso a la totalidad de los pacientes por la condición clínica crítica. En cuanto a las enzimas cardiacas no contamos suministro de kits suficientes para su realización.

La biopsia endimiocárdica para diagnóstico conclusivo en dengue ofrece más dificultades en pacientes con trastornos de la hemostasia como son los niños con fiebre dengue hemorrágico, proponiendo una combinación de herramientas no invasivas como son la clínica, alteraciones electro y ecocardiográficas 
Agradecimientos. A todos los niños que participaron en el estudio y nos permitieron conocer el comportamiento de la enfermedad.

\section{REFERENCIAS}

1. World Health Organization (WHO). Dengue and Dengue Haemorrhagic Fever. Geneva: WHO; 1997.

2. Malavige, S Fernando, D J Fernando, S L Seneviratne. Dengue viral infections, Postgrad Med 2004; 80: 588-601.

3. Gulati S, Maheshwari A. Atypical manifestations of dengue. Trop Med Int Health. 2007; 12(9): 1087-95.

4. Solomon T, Dung, NM, Vaughn DW, Kneen R. Neurological manifestations of dengue infection. Lancet. 2000; 355 (9209):1053-9.

5. Huan-Yao L, Trai-Ming Y, Hsiao-sheng L. Inmunopathogenesis of dengue virus infection. J Biomed Sci 2001; 8:377-388.

6. Yraima L, Valero N, Estévez J. Alteraciones hepáticas en pacientes con dengue. Investigación. Clínica. 2005; 46 (2): 169-78.

7. Ribeiro R, Goncalves H, Oliveiro J, De Souza O. Dengue virus type 3, Brazil, 2002. Emerg Infect Dis 2005; 11: 1376-81.

8. Kamath S, Ranjit S. Clinical features, complications and atypical manifestations of children with severe forms of dengue hemorrhagic fever in South India. The Indinan J Pediatrics 2006; 73: 889-895.

9. Shekerdemian L, Bohn D. Acute viral myocarditis: Epiemiology and pathophysiology. Pediatr Crit Care 2006; 7: s1-s7.

10. Prompan W. Dengue Myocarditis. Southeast Asia J. Trop Med Public Health 2004; 35 (3) 611-3.

11. Chaturvedy UC, Agarwal R, Elbishbishi, Mustafa AS. Cytokines cascade in hemorrhagic fever: implications for pathogenesis. FEMS Immunol Med Microbiol 2000; 28: 183-188.

12. Rothman A. Dengue: defining protective versus pathologic immunity. J Clin Invest 2004; 113: 946-51.

13. Nagaratnam N, Siripala K, Silva N. Arbovirus (dengue type) as a cause of acute myocarditis and pericarditis. British Heart Journal 1972;35: 204-06.

14. Yusoff $\mathrm{H}$. Electrocardiographic and Echocardiographic Changes During the Acute Phase of Dengue Infection in Adults. J HK Coll Cardiol 1993, 1: 93-96.

15. Martinez- Torres E. Dengue y Dengue hemorrágico Aspectos clínicos. Salud Pública Mex. 1995; 37 suppl: $529-44$.

16. Kabra SK, Jain Y, Pandey RM, et al. Dengue haemorrhagic fever in children in the 1996 Delhi epidemic. Trans R Soc Trop Med Hyg 1999;93:294-8.

17. Wali JP, Biswas A, Chandra S, et al. Cardiac involvement in dengue hemorrhagic fever. Int $\mathrm{J}$ Cardiol 1998;64:31-6.

18. Horta Veloso H, Ferreira Junior JA, Braga de Phava JM, Faria Honorio J, et al. Acute atrial fibrillation during dengue hemorrhagic fever. Braz J Infect Dis 2003; 7(6): 418-22.

19. Khongphatthanayothin A, Suesaowalak M, Muangmingsoq S, Bhattarakosol P, Pancharoen Ch. Hemodinamic profiles in patients with dengue hemorrhagic fever during toxic stage: an echocardiographic study. Intens Care Med. 2003;29:570-574.

20. Ranjit S, Kissoon N, Jayakumar I. Aggressive management of dengue shock syndrome may decrease mortality rate: a suggested protocol. Pediatr Med care Med 2005; 6: 412-419.

21. Mendez A, Gonzalez G. Dengue hemorrhagic fever in children: ten year of clinical experience. Biomedica 2003; 23: 180-93.

22. Salgado DM, Rodríguez JA, Garzón M, Cifuentes M Vega M, Castro D. Clinical and epidemiological characterization of hemorrhagic dengue fever in Neiva, Colombia 2004. Rev Salud Publica (Bogotá) 2007; 9(1):53-63. 
23. Shann F. Severe dengue: Coming soon to a pediatric intensive care unit near you?. Pediatr Crit Care Med 2005; 6: 490-91.

24. Ranjit S, Kissoon N, Gandhi D,Dayal A, Rajeshwari N, Kamath S. Early differentiation between dengue and septic shock by comparison of admission hemodynamic, clinical, and laboratory variables. Pediatr Emerg Care. 2007; 23, (6): 368-376.

25. Khongphatthanayothin A, Lertsapcharoen P, Supachokchaiwatana P, La-orkhunV, Khumtonvong A, Boonlarptaveechoke Ch, Pancharoen Ch. Myocardical depresión in dengue hemorrhagic fever: prevalence and clinical descripción. Pediatr Crit Care Med. 2007;8 :1-6.

26. Shrishu K, Suchitra R. Clinical features, complications and atypical manifestation of children with severe forms of dengue hemorrhagic fever in south India- Indian J Pediatr 2006; 73: 889 - 895.

27. Salgado DM, Vega M, Garzón M, Panqueba C, Rodriguez JA. Mortalidad por Dengue Hemorrágico en niños en Colombia: más allá del choque. Infectio 2008; 12: 249-255. 